\title{
Statistical Antenna Proximity Effect Modeling with Uncertainty Impedance Ellipses
}

\author{
Pedro Luis Carro, Jesús de Mingo, Paloma García-Dúcar, and Antonio Valdovinos \\ Department of Electronic Engineering and Communications, Aragon Institute of Engineering Research (I3A), University of Zaragoza, \\ 50018 Zaragoza, Spain \\ Correspondence should be addressed to Pedro Luis Carro; plcarro@unizar.es
}

Received 14 June 2017; Accepted 30 October 2017; Published 13 December 2017

Academic Editor: Symeon Nikolaou

Copyright ( 2017 Pedro Luis Carro et al. This is an open access article distributed under the Creative Commons Attribution License, which permits unrestricted use, distribution, and reproduction in any medium, provided the original work is properly cited.

\begin{abstract}
User effects on portable antennas can decrease the global terminal performance, such as reducing the battery life or the wireless coverage. People presence will modify the input impedance, the far field radiation pattern, the radiation efficiency, and the near field. In this paper, we investigate the influence of the user on the input impedance by means of a new statistical approach which relies on uncertainty, where a handheld antenna works in two modes (data and talk) while people couple to the antenna. Studying some reflection coefficient measurements performed with a vector network analyzer (VNA), a standard framework based on uncertainty ellipses is developed. Finally, the method is applied to some antenna prototypes, showing its advantages and reliability to quantify and compare proximity effects.
\end{abstract}

\section{Introduction}

Mobility and user proximity impact on handheld terminals and radiating structures has become a key issue in order to improve standard portable antenna designs. During the last 20 years, some researchers have focused on the evaluation of the human body impact [1-5], which has been mostly carried out by means of electromagnetic simulations and verified sometimes by measurements.

On the one hand, in spite of the clear general understanding of the head, hands, and even other body parts and surrounding objects on antenna circuit and field parameters, the investigations have been carried out presenting averaged ratios of usual quantities, such as the input impedance $[6,7]$ or the radiation pattern [8]. However, we should not forget that neither the hands nor the user's head keeps the same position relatively to the antenna, and consequently, there is some variability around those averages [9]. It is always possible to study a few feasible configurations, but some magnitudes like the antenna input impedance will be no longer static in real scenarios. Therefore, the actual "instantaneous" input impedance will be near the obtained values for a finite set of suitable states. Even though modern computational electromagnetic techniques can deal efficiently with each state separately, calculating exactly the actual antenna radiation pattern and the input impedance might be difficult.

On the other hand, there is also a growing concern about the final effects on the system where the antenna is integrated, working in transmitting or receiving mode (e.g., the signal to noise ratio (SNR) variations due to antenna and receiver mismatching $[10,11]$ or the degradation on power amplifier (PA) performance). According to those works, the impact of mismatching is not negligible and must be considered so that the communication system can be properly designed. Mismatch may be even more relevant in the case of wireless technologies since there are additional sources of variability introduced by mobility and user interaction with the antenna. In fact, some solutions have been suggested to reduce mismatch, such as impedance tuning networks or reconfigurable antennas [12-14]. They are supposed to reduce the reflection coefficient dynamically by means of variable lumped or adaptive transmission line elements. 


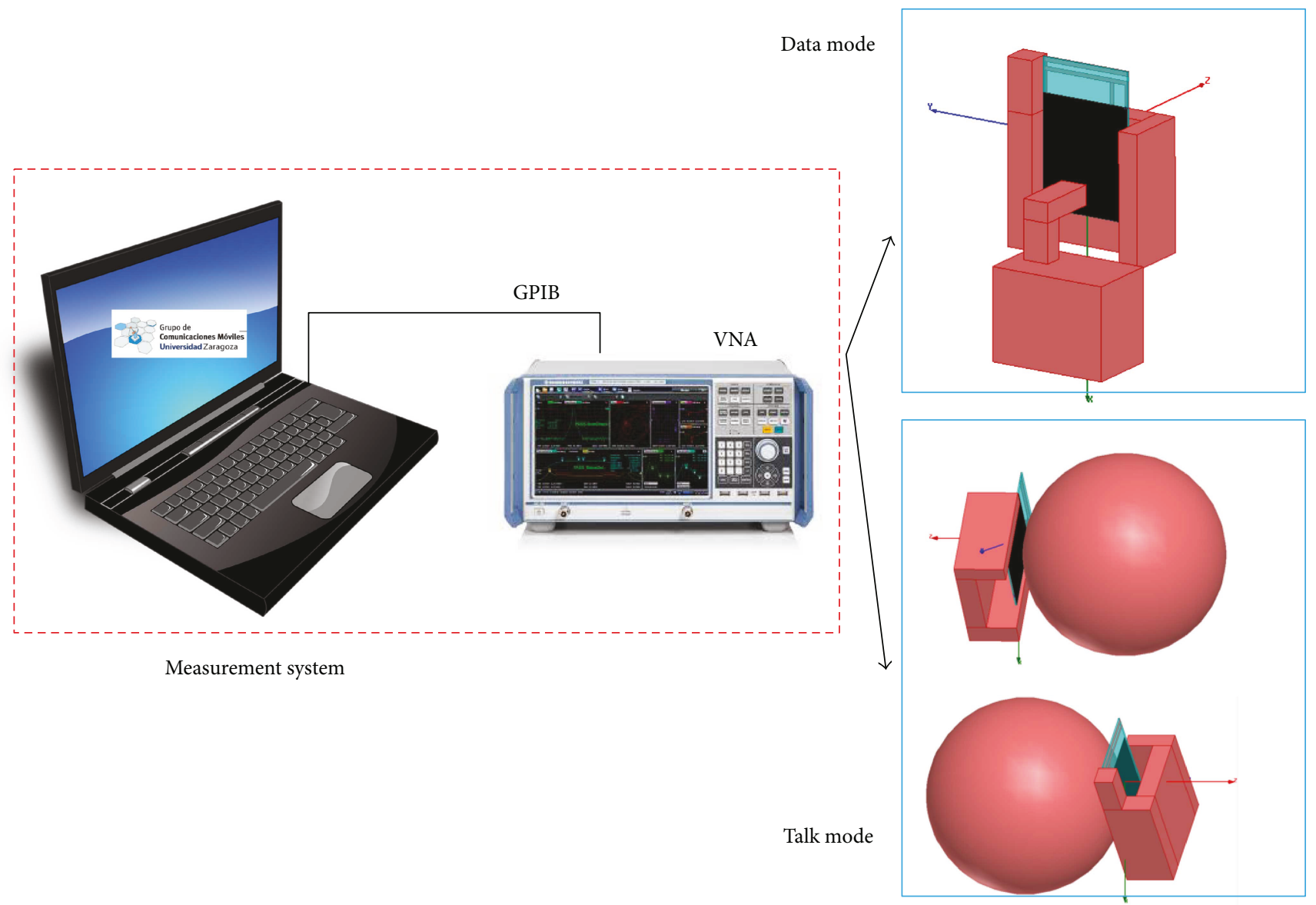

FIgURE 1: Typical antenna coupling modes for wireless applications.

In contrast to other radiating structures, portable antennas should be characterized not only in terms of the classical static magnitudes (input impedance, radiation pattern, axial ratio, etc.) but also from the dynamical point of view. There is a lack of dynamic models in the literature which would be very helpful to quantify the variability and to develop improved portable antennas. In addition, these models could be relevant to design receivers and transmitters because suitable mitigation policies could avoid a bad reception and improve the battery life or the handheld coverage.

The proposed novel approach analyzes the impedance variability. It is not exactly a dynamical model but aims at estimating the impedance variability region on the Smith chart and is based on statistical measurements. The advantage of the method relies on its generality since it can be applied to any antenna geometry, object, and frequency band. It allows to compare portable antenna performances and, moreover, provides to the antenna and microwave engineer a procedure to build the variability region with a few parameters in order to evaluate the performance degradation or to simplify and improve impedance tuning networks or reconfigurable circuit designs.

The outline of the paper is as follows. First, we establish the connection between proximity effects and variability by means of the support of the probability density function
(PDF) corresponding to the reflection coefficient. Second, we estimate the variability with the aid of the uncertainty ellipses which come naturally from the covariance matrix corresponding to the experimental measurements accomplished to study the effect of certain objects. We focus on a typical and practical mobile scenario with real antennas in the UTRA/FDD LTE B8 and UTRA/FDD LTE B3 frequency bands. The ellipse framework requires five geometrical parameters which may be estimated with certain confidence. We present the evaluation of this new method performed on three prototypes and give the meaning of those parameters in terms of the variability. Finally, we propose the 95\% ellipse as its measure and some applications on reconfigurable tuning networks.

\section{Statistical Approach to the Impedance Variation Problem}

Figure 1 shows a typical wireless terminal where the antenna is characterized as a complex input impedance from the circuit point of view and the interaction with a user in two possible modes (talk and data transmission configurations). The input impedance (at the frequency $\omega_{0}$ ) is denoted as $Z_{a}(\omega)=R_{a}\left(\omega_{0}\right)+j X_{a}\left(\omega_{0}\right)$, where $R_{a}$ is the resistance and $X_{a}$ is the reactance. 
Antenna proximity effects can be geometrically identified on the Smith chart. Dynamic coupling transforms the point which represents the static impedance at a fixed frequency onto a set of probable impedance values which constitute a variability area. Thus, some dynamic coupling features can be analyzed by studying and quantifying this Smith chart subdomain. Identically, the input impedance curve that models the antenna frequency band behavior is also transformed into a certain area, and thus, similar techniques can be applied to broadband or narrowband problems.

2.1. Variability Impedance Region through the Reflection Coefficient in the Matching Domain. Let a radiating structure be placed close to the user in a wireless environment working at the frequency $\omega_{0}$. The antenna will have an input impedance $Z_{a}(n)$ at the time $n$. Then, its instantaneous reflection coefficient is defined by

$$
\Gamma(n)=\frac{Z_{a}(n)-Z_{0}}{Z_{a}(n)+Z_{0}}=\frac{\bar{Z}_{n}^{a}-1}{\bar{Z}_{n}^{a}+1,}
$$

where $Z_{0}=50 \Omega$ is the reference impedance and $\bar{Z}^{a}$ is the normalized antenna impedance. The modeling process can be carried out either on the antenna input impedance or on the instantaneous reflection coefficient, because both reflect the same concept, but in different domains. They are complex random variables in discrete time $(n)$.

In order to keep the input impedance study as simple as possible, it is important to find out which is the most convenient representation. There are four different possibilities, but the Cartesian form where the reflection coefficient is mathematically represented by its real and imaginary parts $\Gamma_{n}=\lambda_{n}+j \mu_{n}$, being that $\lambda$ and $\mu$ are real numbers, is preferred because the components of the random vector

$$
\Gamma_{n} \equiv\left(\lambda_{n}, \mu_{n}\right)-1<\lambda_{n}<1,-1<\mu_{n}<1
$$

have identical compact support and dimensional units. In addition, it is easy to estimate the input impedance from

$$
\bar{Z}_{n}=\frac{1+\Gamma_{n}}{1-\Gamma_{n}}
$$

where $\bar{Z}$ denotes the normalized impedance.

Rigorously, the reflection random vector which models the impedance variations is a stochastic process in discrete time. The cross-correlation, covariance, or higher order moments are typically required to describe its properties from a mathematical point of view. However, if antenna designers only need to compute the main magnitudes and do not deal with prediction issues in time, the probability density of $\Gamma \equiv(\lambda, \mu)$ can be accurate enough.

We define mathematically the impedance variability region (IVR), $\Omega_{Z}$, as the closure of the set of possible values of $(\lambda, \mu)$ having the density $f_{(\lambda, \mu)}(x, y)$ on the Smith chart space $\mathbb{S C}$ such that

$$
\Omega_{Z}=\left\{\frac{\Gamma \in \mathbb{S C}}{f_{(\lambda, \mu)}(x, y)}>0\right\} .
$$

The impedance variability region (whose limit is the Smith chart circle $|\Gamma|=1$ ) contains the proximity effects and depends on the antenna geometry and the nature of the interaction, hence the interest of estimating such a region with certain confidence. As mentioned, it can be applied to a single frequency or to a complete frequency band.

2.2. $\Omega_{Z}$ : Statistical Estimation. A VNA can collect a set of reflection coefficient samples from an antenna every $T_{m}$ second. The measurement process is performed while the antenna is moving on a certain path or, for example, simulating a phone call with the radiating structure close to the user's head or pocket. The coupling phenomena appear in these data and will be very different from the static values.

The sampling time (or sampling frequency) depends on the VNA capabilities and the frequency of interest. Once the vector samples are measured $\left(\boldsymbol{\Gamma}_{1}, \ldots, \boldsymbol{\Gamma}_{N}\right)$, the estimated impedance variability region $\widehat{\Omega}_{Z}$ may be calculated from the approximated probability density of $\Gamma \equiv(\lambda, \mu)$.

The samples obtained after the data registration can be arranged in a complex vector

$$
\underline{\Gamma}=\left(\lambda_{1}+j \mu_{1}, \lambda_{2}+j \mu_{2}, \ldots, \lambda_{N}+j \mu_{N}\right),
$$

which can be partitioned into a number of equal-sized cells of the area $d x \times d y$ because the support of the random components is compact $([-1,1] \times[-1,1])$. The density estimation $\widehat{f}(\lambda, \mu)$ at a point $(x, y)[15]$ is

$$
\widehat{f}_{(\lambda, \mu)}(x, y)=\frac{n_{j}}{\sum_{j} n_{j} d x d y},
$$

where $n_{j}$ is the number of samples in the $j$ th cell, called bin. According to the approximated numerical estimation, $\widehat{\Omega}_{Z}$ is

$$
\widehat{\Omega}_{Z}=\left\{\frac{\Gamma \in \mathbb{S C}}{\widehat{f}_{(\lambda, \mu)}(x, y)}>0\right\} .
$$

For example, the impedance variations are very small when the antenna is isolated. As a result, the impedance density should tend to a Dirac distribution

$$
P\left(\lambda=\lambda_{0} \cap \mu=\mu_{0}\right)=\delta\left(\lambda-\lambda_{0}\right) \delta\left(\mu-\mu_{0}\right),
$$

and the estimated impedance variability region should approximate to

$$
\widehat{\Omega}_{Z}=\left\{\frac{\Gamma \in \mathbb{S C}}{\widehat{f}_{(\lambda, \mu)}(x, y)}>0\right\} \rightarrow\left(\lambda_{0}, \mu_{0}\right) .
$$

\section{Reflection Coefficient Statistical Measurements under User Influence}

3.1. Method. The computation of the approximated impedance variability region begins with the experimental antenna measurement sampling (Figure 2(a)). There are many feasible situations where an impedance fluctuation analysis is important. Since the complex environment includes so many objects interacting with the antenna, we reduce the research 


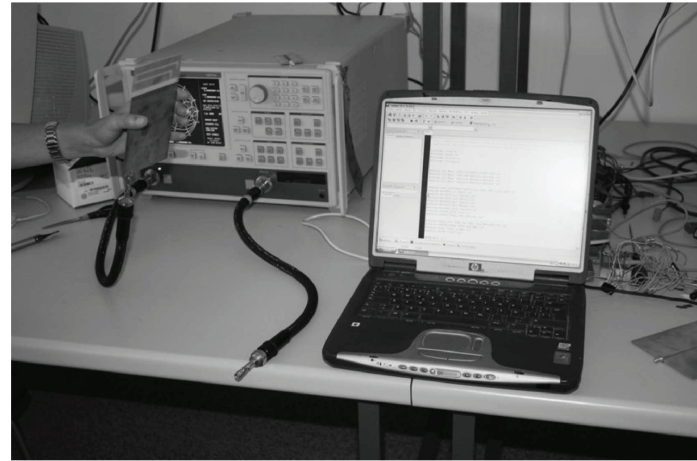

(a)

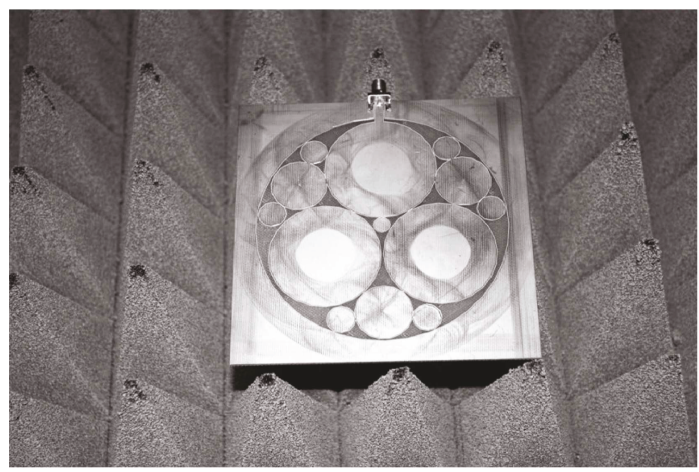

(c)

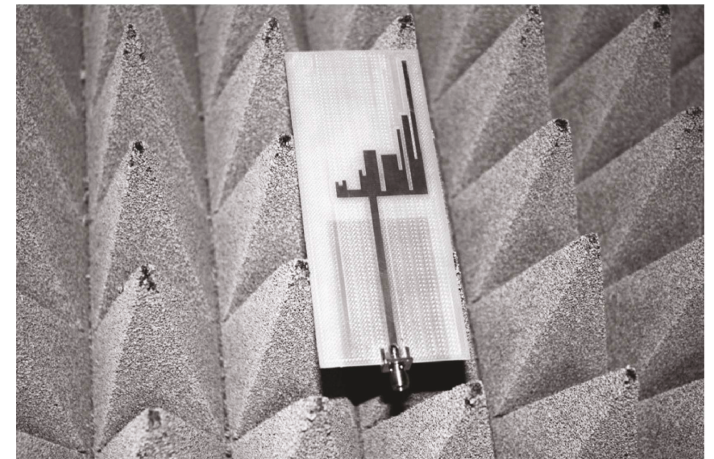

(b)

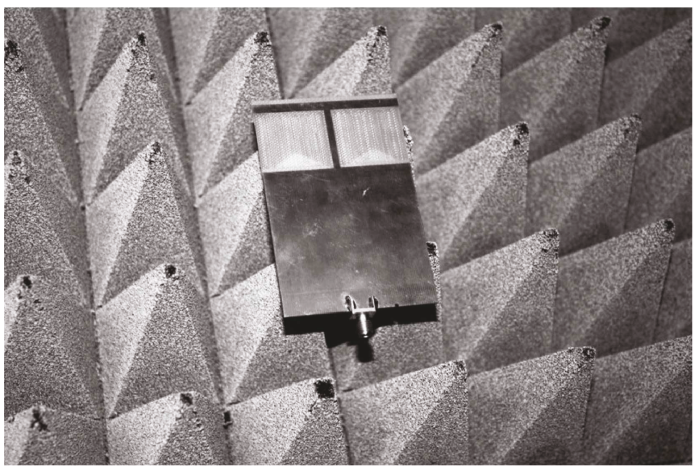

(d)

Figure 2: Setup elements. (a) Details of the hand and the capture system (PC + GPIB + VNA). (b) Single-sided fractal antenna (Cantor model). (c) CPW-fed printed slot antenna (Descartes model). (d) Double-sided printed antenna (T-monopole model).

in the present work to a set of the most probable scenarios and study the radiating structure proximity effect in two situations: the so-called "data" and "talk" modes. When the terminal is operating in a "data mode," the antenna is held by a user close to the body to simulate the access to internet applications. When the user is performing a phone call, the geometry is held with the hand near the head and this is the "talk mode." Besides, the user has certain mobility around to include the dynamic effect. The VNA (Anritsu 37000) captures the reflection coefficient while the person performs his tasks and registers the interaction.

As far as the frequency band is concerned, the study is carried out in the UTRA/FDD LTE B8 and UTRA/FDD LTE B3 frequency bands. This aims at showing the proposed method advantages in real applications, but the approach is applicable to other frequency bands as well. The measurement process is made by means of in-house software which samples the instantaneous reflection coefficient through a GPIB device. The maximum sampling frequency depends on the VNA and the equipment calibration. In this work, data have been collected using a $1 \mathrm{~ms}$ sampling time. The user dynamics is very slow compared to the signal frequency, and this sampling frequency can record most of the proximity effects.

3.2. Antennas under Test, Experimental Setup, and Results. Figures 2(b)-2(d) show the test configurations to capture the reflection coefficient variability. Three different antennas [16-18] suitable for medium-size mobile devices have been designed on FR4 substrate: a single-sided fractal antenna (Cantor model, Figure 2(b)), a CPW-feed printed slot antenna (Descartes model, Figure 2(c)), and a doublesided printed antenna (T-monopole model, Figure 2(d)). The prototypes are intended to work according to the UTRA/FDD LTE B8 and UTRA/FDD LTE B3 standard. The requirement concerning the return losses is $6 \mathrm{~dB}$, but they have different scattering parameters $\left(S_{11}\right)$ in those frequency bands. The antennas differ in field and directivity performance because of the current paths on the conductors, in spite of the underlying radiation physics similarity (the structures are based on printed technology). Figure 3 presents the experimental reflection coefficient and points out the differences between the prototypes.

Three experiments are carried out to measure the reflection coefficient magnitude and its phase in independent realizations. The dynamical reflection coefficient is captured for every frequency of interest in three situations. The antenna is placed inside the anechoic chamber and works under no proximity effects in the static case. Each recording contains 5000 points because the variability should be very low. Data and talk modes are measured when the user interacts with the radiating structure during 4 minutes approximately, which turns out in 25000 samples for each frequency. The UTRA/FDD LTE B8 and UTRA/ 


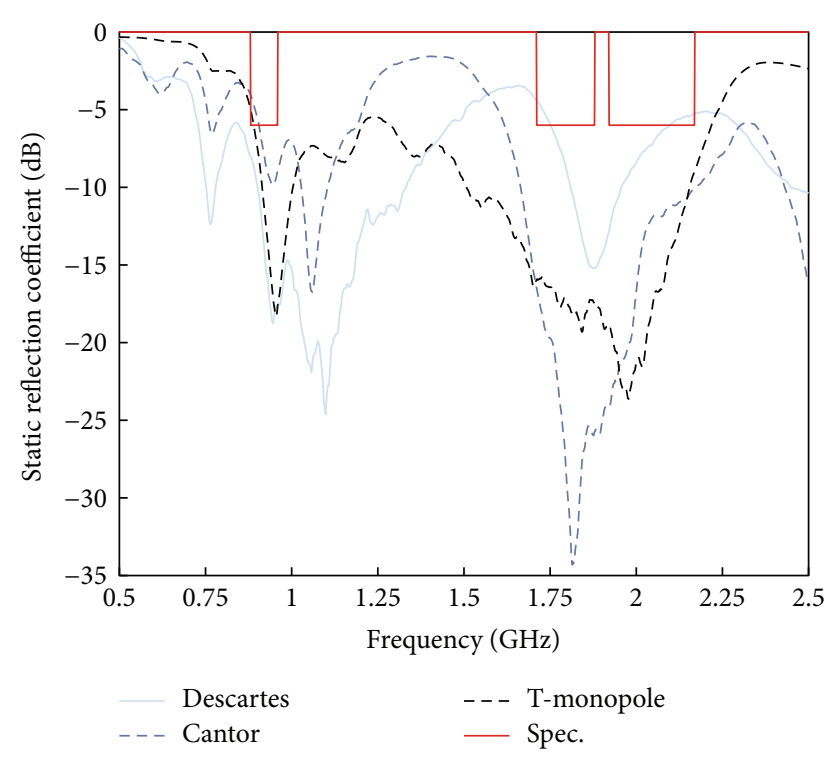

FIgURE 3: Static reflection coefficient of the antenna test-beds.

FDD LTE B3 bands [19] are tested in the up- and downlink operative frequencies:

(i) UTRA/FDD LTE B8 uplink (UL) test frequencies: $880,897.5$, and $915 \mathrm{MHz}$

(ii) UTRA/FDD LTE B8 downlink (DL) test frequencies: $925,942.5$, and $960 \mathrm{MHz}$

(iii) UTRA/FDD LTE B3 uplink (UL) test frequencies: $1710,1747.5$, and $1785 \mathrm{MHz}$

(iv) UTRA/FDD LTE B3 downlink (DL) test frequencies: $1805,1842.5$, and $1880 \mathrm{MHz}$

Finally, the samples are mixed to estimate the total inband variability region, which is of interest, for example, to determine the required coverage area for an adaptive antenna matching network or to compute the element values in reconfigurable antenna structures. Unlike the isolated antenna, with very small variations, the matching domain representation states the impedance range as a result of the user interaction, as shown in Figure 4 in the particular case of the Descartes model.

In fact, Figure 4 points out the difference in the measured scenarios. Both UTRA/FDD LTE B8 and UTRA/FDD LTE B3 impedances are clearly observed at the six corresponding frequencies. These curves spread to the variability regions in data or talk modes, and the final dynamical impedance region is their ensemble. Furthermore, the data distributions are not uniformly spread since not all the measurements have the same probability. These PDFs depend on the frequency and the antenna geometry. According to the measurements, the variability decreases when the frequency increases due to a stronger coupling in lower bands (the user is closer in terms of wavelength in the UTRA/FDD LTE B8 standard).

Even though the impedance variability region can be estimated from the measured histogram, the impedance region is strongly dependent on the measured reflection coefficient PDF which is a disadvantage because the comparison between different antennas can be unavailable. Thus, it may be more reliable for engineers to seek a statistical alternative with few parameters which is applicable to any radiating structure.

\section{Theoretical Analysis Framework: Uncertainty Impedance Ellipses}

The proposed general framework is based on the analysis of the uncertainty [20-22] in the reflection coefficient. The study of the impedance PDF support has defined a nonuniform area $\left(\Omega_{Z}\right)$, which is different even for the same antenna operating as a multiband structure. We propose to approximate $\Omega_{Z}$ by means of an impedance ellipse, which may be a convenient method to express the uncertainty of the antenna input impedance in a graphical format.

If the observed reflection coefficients are referred to the Cartesian coordinate system on the $\mathbb{S C}$ space, they can be written in a vector notation as

$$
\Gamma_{\mathbf{i}}=\left[\begin{array}{l}
\lambda_{i} \\
\mu_{i}
\end{array}\right]
$$

Then, we could calculate the estimated mean $\overline{\boldsymbol{\Gamma}}$ corresponding to $N$ samples as

$$
\overline{\boldsymbol{\Gamma}}=\frac{1}{N} \sum_{i=1}^{N} \boldsymbol{\Gamma}_{\mathbf{i}}=\frac{1}{N}\left[\begin{array}{c}
\sum_{i} \lambda_{i} \\
\sum_{i} \mu_{i}
\end{array}\right]=\left[\begin{array}{l}
\bar{\lambda} \\
\bar{\mu}
\end{array}\right] .
$$

The estimated covariance matrix $\widehat{\Sigma}$ should be computed as

$$
\widehat{\boldsymbol{\Sigma}}=\left(\begin{array}{cc}
\sigma_{\lambda}^{2} & \rho \sigma_{\lambda} \sigma_{\mu} \\
\rho \sigma_{\mu} \sigma_{\lambda} & \sigma_{\mu}^{2}
\end{array}\right)=\frac{1}{N-1} \sum_{i=1}^{N}\left(\boldsymbol{\Gamma}_{i}-\overline{\boldsymbol{\Gamma}}\right)\left(\boldsymbol{\Gamma}_{i}-\overline{\boldsymbol{\Gamma}}\right)^{\mathrm{T}}
$$

where $\sigma_{\lambda}$ and $\sigma_{\mu}$ denote the standard deviations corresponding to the real part $\lambda$ and the imaginary part $\mu$ of the reflection coefficient and $\rho$ is the correlation index for $\lambda$ and $\mu$. The quadratic form

$$
(\boldsymbol{\Gamma}-\overline{\boldsymbol{\Gamma}})^{\mathrm{T}} \widehat{\Sigma}^{-1}(\boldsymbol{\Gamma}-\overline{\boldsymbol{\Gamma}})=\ell^{2}
$$

is the probability impedance ellipse and an approximation of $\Omega_{Z}$. A certain percentage of the actual impedance values (depending on the statistical distribution) is within the ellipse. Typically, $\ell$ is chosen in terms of the standard deviation $\sigma$. If $\ell=1,2$, or 3 , these are called $1 \sigma, 2 \sigma$, and $3 \sigma$ impedance ellipses. If this parameter increases, the ellipses have larger axes and more impedance values are contained inside. We will discuss a method to choose this value in the following section, but the idea is that it suggests a confidence bound which depends on the statistical distribution. 


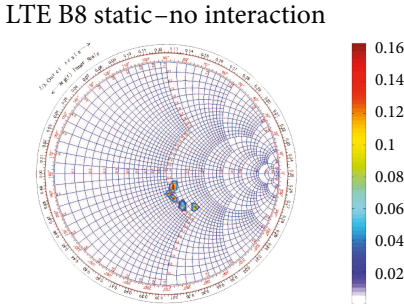

LTE B8 dynamic-data mode

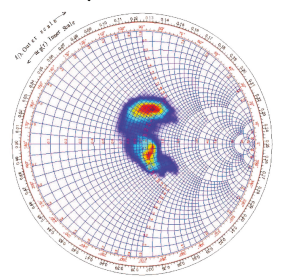

LTE B8 dynamic-talk mode

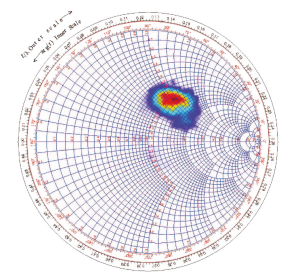

LTE B8 final coverage area

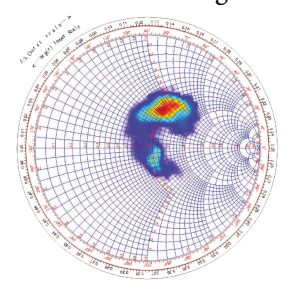

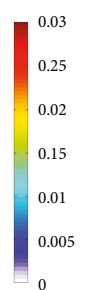

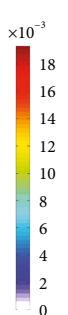

(a)

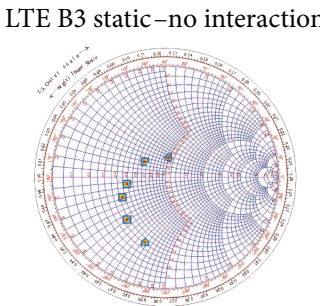

LTE B3 dynamic-data mode

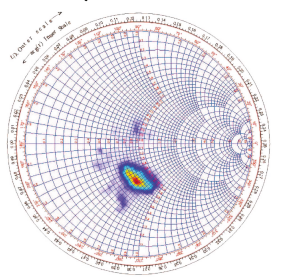

LTE B3 dynamic-talk mode
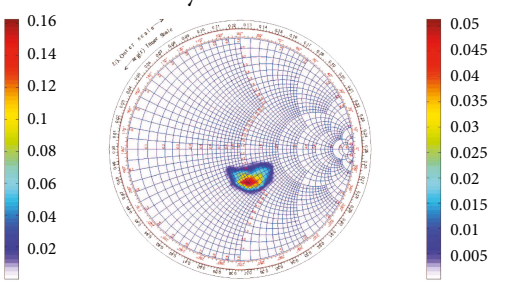

LTE B3 Final coverage area
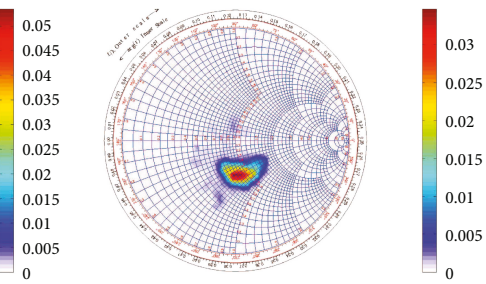

(b)

FIGURE 4: Experimental Smith chart representation of the reflection coefficient samples for Descartes model. (a) UTRA/FDD LTE B8 band. (b) UTRA/FDD LTE B3 band.

The impedance ellipse can cast as

$$
\frac{1}{1-\rho^{2}}\left[\frac{(\lambda-\bar{\lambda})^{2}}{\sigma_{\lambda}^{2}}+\frac{(\mu-\bar{\mu})^{2}}{\sigma_{\mu}^{2}}-2 \rho \frac{(\lambda-\bar{\lambda})(\mu-\bar{\mu})}{\sigma_{\lambda} \sigma_{\mu}}\right]=\ell^{2}
$$

containing the information on the dynamic coupling and proximity effects caused by the user. The main advantage of the proposed method is that coupling effects can be viewed with a novel geometric perspective. Furthermore, only five geometrical parameters are required for modeling: the ellipse center, the major and minor axis, and the rotation angle (related to the $\mathbb{S C}$ space axes [23], as Figure 5 shows). Therefore, the antenna engineer, with the knowledge of these five magnitudes, is able to build an approximate variability region with a certain confidence level, which can be used for developing proper matching networks or evaluating antenna performance.

The algorithm to compute those five geometrical parameters relies on the analysis of the statistical data.

4.1. Ellipse Center. Compute the ellipse center and the first and second values, by using (11).

4.2. Principal Axes. Calculate the principal axes with the aid of the $\widehat{\boldsymbol{\Sigma}}$ matrix. Notice that we can perform a single-value decomposition (SVD) on $\widehat{\boldsymbol{\Sigma}}$ to obtain two eigenvalues, $\gamma_{1}$ and $\gamma_{2}$, and two eigenvectors, $\tilde{\lambda}$ and $\tilde{\mu}$, so that

$$
\mathbf{D}=\mathbf{U}^{\mathrm{T}} \widehat{\boldsymbol{\Sigma}} \mathbf{U}
$$

where $\mathbf{D}$ and $\mathbf{U}$ are the diagonal and orthogonal matrices whose columns are the eigenvectors such that

$$
\begin{aligned}
& \mathbf{D}=\left(\begin{array}{ll}
\gamma_{1} & 0 \\
0 & \gamma_{2}
\end{array}\right), \\
& \mathbf{U}=\left(\begin{array}{ll}
\cos \Psi & -\sin \Psi \\
\sin \Psi & \cos \Psi
\end{array}\right) .
\end{aligned}
$$

The principal axes are given by

$$
\begin{aligned}
& \tilde{\mathbf{e}}_{\lambda}=\left[\begin{array}{l}
\cos \Psi \\
\sin \Psi
\end{array}\right], \\
& \tilde{\mathbf{e}}_{\mu}=\left[\begin{array}{c}
-\sin \Psi \\
\cos \Psi
\end{array}\right],
\end{aligned}
$$

where $\Psi$ is the rotation angle of the ellipse (third parameter).

4.3. $\ell \sigma:$ Major and Minor Axes. The fourth and fifth parameters are computed which provided a specified value of $\ell$. Assuming that $\gamma_{1}$ is the maximum eigenvalue,

$$
\begin{aligned}
& a=\sqrt{\ell^{2} \gamma_{1}}, \\
& b=\sqrt{\ell^{2} \gamma_{2}} .
\end{aligned}
$$

To sum up, our approach evaluates the proximity user effect through the uncertainty impedance ellipse with parametric boundary where $t \in[0,2 \pi]$ is a variable which generates the ellipse contour $\partial \Omega_{Z}$

$$
\partial \Omega_{Z} \approx\left\{\begin{array}{l}
\lambda(t)=\bar{\lambda}+a \cos t \cos \Psi-b \sin t \sin \Psi, \\
\mu(t)=\bar{\mu}+a \cos t \sin \Psi+b \sin t \cos \Psi .
\end{array}\right.
$$

\section{Antenna Evaluation and Results}

The proposed evaluation method is applied to the UTRA/ FDD LTE B8 and UTRA/FDD LTE B3 antenna prototypes 


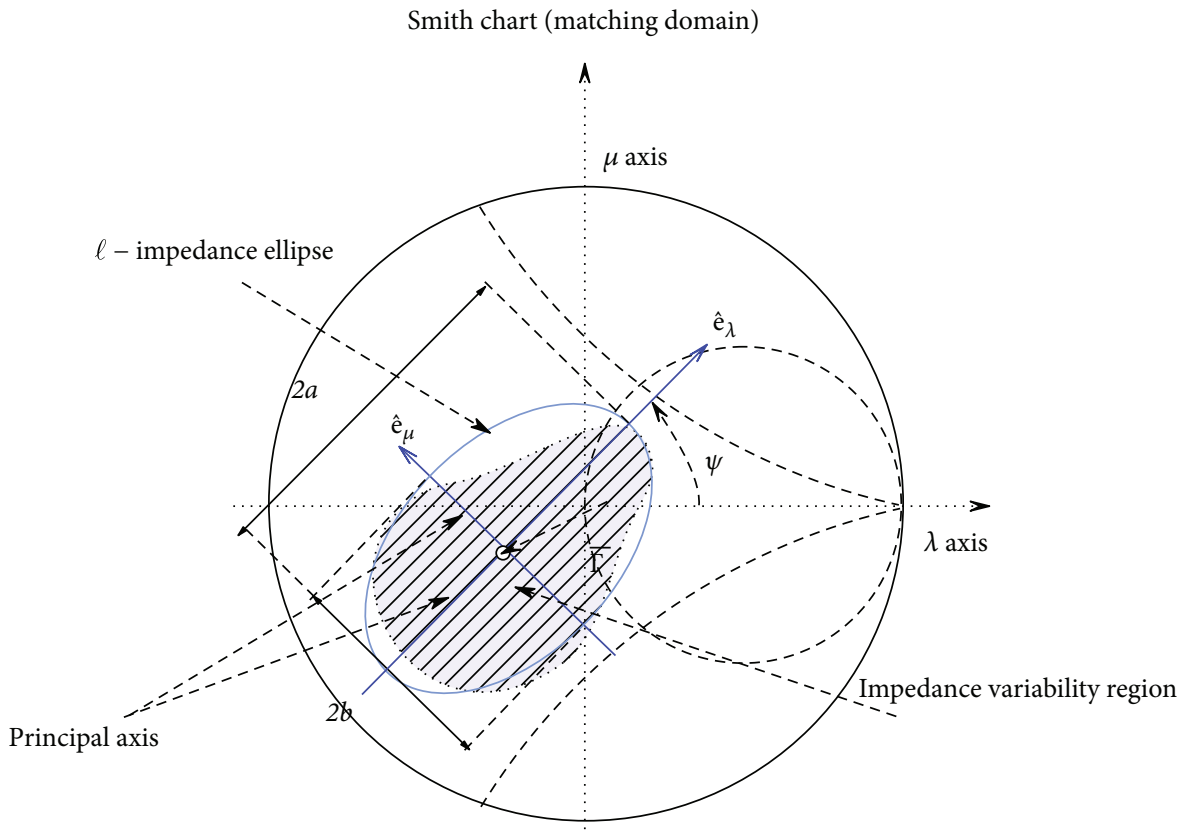

FIGURE 5: Impedance variability description with the ellipsoidal geometry and its five parameters.

TABLE 1: Uncertainty impedance ellipse parameters and confidence levels.

\begin{tabular}{|c|c|c|c|c|c|c|c|c|c|c|c|c|c|}
\hline Antenna & Band & Scenario & $\gamma_{1}$ & $\gamma_{2}$ & $\Psi$ & $\bar{\lambda}$ & $\bar{\mu}$ & $1 \sigma$ & $2 \sigma$ & $3 \sigma$ & $k \sigma(95 \%)$ & $a$ & $b$ \\
\hline \multirow{6}{*}{ Cantor } & \multirow{3}{*}{ UTRA/FDD LTE B8 } & $\mathrm{D}+\mathrm{T}$ & 0.0262 & 0.0097 & 19.81 & 0.0552 & 0.1685 & 35.42 & 88.94 & 98.89 & 2.404 & 0.389 & 0.236 \\
\hline & & Talk & 0.026 & 0.0083 & 7.21 & -0.0067 & 0.1275 & 37.09 & 85.48 & 99.37 & 2.404 & 0.387 & 0.219 \\
\hline & & Data & 0.0171 & 0.0094 & 22.99 & 0.1171 & 0.2094 & 33.4 & 88.8 & 98.6 & 2.424 & 0.317 & 0.234 \\
\hline & \multirow{3}{*}{ UTRA/FDD LTE B3 } & $\mathrm{D}+\mathrm{T}$ & 0.0067 & 0.0047 & -57.3 & 0.0137 & -0.1125 & 39.44 & 88.15 & 98.18 & 2.485 & 0.203 & 0.169 \\
\hline & & Talk & 0.0057 & 0.0026 & 42.34 & 0.0394 & -0.144 & 35.25 & 87.71 & 98.95 & 2.353 & 0.178 & 0.121 \\
\hline & & Data & 0.0073 & 0.0037 & -54.96 & -0.0119 & -0.0807 & 41.79 & 87.49 & 98.27 & 2.484 & 0.213 & 0.15 \\
\hline \multirow{6}{*}{ Descartes } & \multirow{3}{*}{ UTRA/FDD LTE B8 } & $\mathrm{D}+\mathrm{T}$ & 0.0394 & 0.016 & 68.9 & 0.1318 & 0.2281 & 33.6 & 87.7 & 99.68 & 2.313 & 0.459 & 0.293 \\
\hline & & Talk & 0.013 & 0.012 & 81.038 & 0.218 & 0.3319 & 47.2 & 90.87 & 96.49 & 2.424 & 0.277 & 0.268 \\
\hline & & Data & 0.038 & 0.01 & -85.177 & 0.0456 & 0.1243 & 27.17 & 90 & 99.7 & 2.252 & 0.441 & 0.234 \\
\hline & \multirow{3}{*}{ UTRA/FDD LTE B3 } & $\mathrm{D}+\mathrm{T}$ & 0.0146 & 0.0117 & -2.137 & 0.0257 & -0.2718 & 53.52 & 87.46 & 94.58 & 3.067 & 0.371 & 0.332 \\
\hline & & Talk & 0.0078 & 0.0031 & 7.4496 & 0.0941 & -0.2618 & 34.74 & 87.68 & 99.29 & 2.394 & 0.212 & 0.133 \\
\hline & & Data & 0.0206 & 0.0117 & -76.8676 & -0.0427 & -0.2802 & 56.88 & 83.08 & 95.75 & 2.939 & 0.422 & 0.317 \\
\hline \multirow{6}{*}{ T-Monopole } & \multirow{4}{*}{ UTRA/FDD LTE B8 } & $\mathrm{D}+\mathrm{T}$ & 0.0448 & 0.0211 & -70.69 & 0.019 & -0.2021 & 49.99 & 84.56 & 96.81 & 2.798 & 0.592 & 0.406 \\
\hline & & Talk & 0.0236 & 0.0123 & 70.04 & 0.0517 & -0.2468 & 50.19 & 85.69 & 97.1 & 2.71 & 0.417 & 0.301 \\
\hline & & Data & 0.0654 & 0.0245 & -65.16 & -0.0124 & -0.1574 & 39.58 & 86.05 & 97.93 & 2.656 & 0.679 & 0.416 \\
\hline & & $\mathrm{D}+\mathrm{T}$ & 0.0321 & 0.0242 & 45.82 & 0.0748 & -0.129 & 48.36 & 86.27 & 97.14 & 2.666 & 0.477 & 0.415 \\
\hline & \multirow[t]{2}{*}{ UTRA/FDD LTE B3 } & Talk & 0.0347 & 0.0252 & 35.8 & 0.067 & -0.1742 & 45.29 & 86.75 & 97.75 & 2.394 & 0.446 & 0.38 \\
\hline & & Data & 0.027 & 0.0216 & 42.3 & 0.0825 & -0.0833 & 57.71 & 85.91 & 94.56 & 3.055 & 0.502 & 0.449 \\
\hline
\end{tabular}

in order to show how the impedance ellipse fits the variability region. The algorithm estimates the geometrical parameters in the multiband dynamic situations (using the ensemble of individual frequencies). Table 1 shows the results for those parameters.

The uncertainty impedance ellipse contains more points when the $\ell$ factor increases according to Figures 6 and 7. In fact, almost all the observations are included at $\ell=3$.
The geometric values allow obtaining different comparisons and effects on the antennas as well as the approximate IVR. As far as the meaning of each parameter is concerned, we realize that the larger the impedance ellipse area, the higher the covariance matrix eigenvalues. This comes out as a result of more influence of the surrounding objects on the radiating structures. Therefore, the eigenvalue product, proportional to the ellipse surface, is a measure of the impedance spreading. 


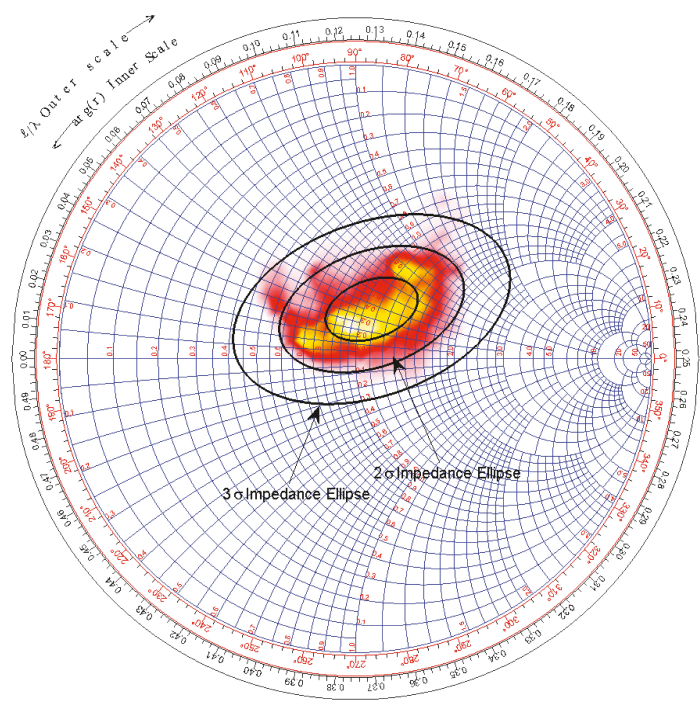

FIgURe 6: $1 \sigma, 2 \sigma$, and $3 \sigma$ ellipses for the Cantor antenna in UTRA/FDD LTE B8 band.

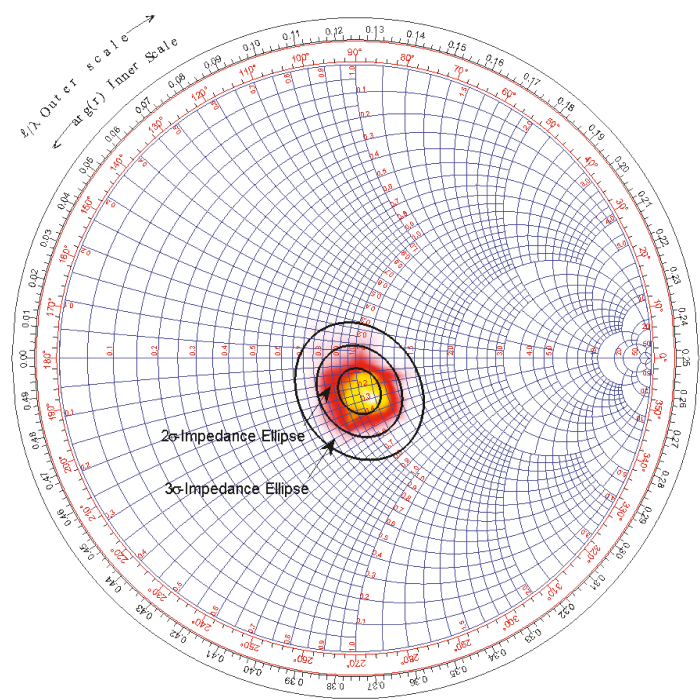

Figure 7: $1 \sigma, 2 \sigma$, and $3 \sigma$ ellipses for the Cantor antenna in UTRA/FDD LTE B3 band.

The ellipse angle is related to the correlation between the antenna resistance and the reactance because it addresses the direction of the main axis, being close to the most usual impedance values. Thus, a positive angle suggests that random changes increasing the antenna resistance tend to result in a reactance displacement towards the inductive Smith chart region (i.e., either reducing the capacitance or increasing the inductance) whereas negative values state the opposite. As the deep connection between the reactance and the near radiated field is well established, we believe the ellipse rotation is strongly influenced by the geometry because it is measuring the reactance sensitivity which provided a change in the resistance (e.g., if $\Psi=0$, only the resistance is affected by the user). However, we should also notice if the eigenvalues are very close, the ellipse is approximately a circumference and, thus, the rotation angle is meaningless.

Finally, the ellipse center is the averaged reflection coefficient which locates the variability region inside the Smith chart. This value may not be the statistical mode (the most frequent reflection coefficient value) because the impedance distribution might not be symmetrical or could be bimodal like in the case of the Descartes antenna in the UTRA/FDD LTE B8 frequency band. The ellipse center can be cast as the averaged dynamic impedance. Its nature (capacitive or inductive) takes into account the fact that the dynamic antenna impedance is more spread in the capacitive or inductive Smith chart subspace.

The proximity effects are compared in terms of three aspects according to the interpretation of the ellipse geometry parameters:

(i) Influence of the frequency band: the eigenvalues are higher in the UTRA/FDD LTE B8 frequency band, and proximity effects are more severe, as expected, at lower frequencies. Since the ellipse surface is a measure of the variability, we define the following factor of merit:

$$
F_{B}=\sqrt{\frac{\gamma_{1}^{B 8} \gamma_{2}^{B 8}}{\gamma_{1}^{B 3} \gamma_{2}^{B 3}}}
$$

The evaluation results are $2.84,1.92$, and 1.11 corresponding to the Cantor, Descartes, and T-monopole structures, respectively.

(ii) Antenna geometry effects: instead of taking into account the multiband aspects, the same idea may be applied to evaluate the structure influence, defining a geometric figure of merit as

$$
F_{G}=\sqrt{\frac{\gamma_{1}^{\text {ant }_{i}} \gamma_{2}^{\text {ant }_{i}}}{\gamma_{1}^{\text {ant }_{j}} \gamma_{2}^{\text {ant }_{j}}} .}
$$

For example, according to the numerical results regarding the UTRA/FDD LTE B8 frequency band $\left(F_{\text {Cantor,Descartes }}=0.69, \quad F_{\text {Cantor,T-monopole }}=0.55\right.$, and $F_{\text {Descartes,T-monopole }}=0.81$ ), the T-monopole is more affected by the user proximity than the Cantor structure. This conclusion remains also valid in the UTRA/FDD LTE B3 frequency band.

(iii) Scenario dependence: data and talk modes have strong differences in their impedance ellipses. We have found that talk mode variations are usually smaller than data mode variations. This result is reasonable due to the user mobility under a phone call regime, which is less than in the data mode (the head dynamics is not as high as around the body). The final uncertainty impedance ellipse (ensemble of the two scenarios, named "datatalk" or " $\mathrm{D}+\mathrm{T}$ ") may be inside, intersecting, or completely outside the data ellipse. In the first case, the trend is that it is slightly smaller than the data 
ellipse. Unlike the inside case, if the data-talk ellipse is outside; its size is bigger than in data scenarios. The talk mode rotation angle is always positive, suggesting displacements towards the inductive Smith chart region when the user effects increase the antenna resistance.

\section{Confidence Level Analysis: $95 \%$ Uncertainty Ellipse}

The uncertainty impedance ellipse is a conic curve which depends on the $\ell$ parameter. The proximity effects may be analyzed by studying the covariance matrix, but some final variability region is required when designers deal with matching issues. We propose to estimate the final IVR by a confidence analysis, that is, the number of observed values which is contained by $\widehat{\Omega}$.

The confidence is estimated by counting the number of observations inside the $\ell$ ellipse. This number depends on the reflection coefficient distribution. If the data were Gaussian, the $\ell$ value could be found relying on the $\chi^{2}$ distribution. However, the impedance PDF can be clearly far from Gaussian and a method to approximate the confidence must be developed.

The following function counts the number of observations:

$$
\eta_{i}\left(\boldsymbol{\Gamma}_{i}\right)= \begin{cases}1, & \text { if } \boldsymbol{\Gamma}_{i} \in \Omega_{Z}^{\ell \sigma}, \\ 0, & \text { otherwise }\end{cases}
$$

where the condition if $\boldsymbol{\Gamma}_{i} \in \Omega_{Z}^{\ell \sigma}$ is equivalent to

$$
\operatorname{dist}\left(\boldsymbol{\Gamma}_{i}, F_{1}\right)+\operatorname{dist}\left(\boldsymbol{\Gamma}_{i}, F_{2}\right) \leq 2 a
$$

being that $F_{1}$ and $F_{2}$ are the ellipse foci and $2 a$ is the major $\ell$ ellipse axis. Finally, the confidence $p$ is then estimated as

$$
p(\ell) \approx \frac{\sum_{i=1}^{N} \eta_{i}}{N} .
$$

Table 1 shows (columns from $1 \sigma$ to $3 \sigma$ ) the estimated confidence for every evaluated case and the $\sigma$ value corresponding to a $95 \%$ confidence (the $k \sigma$ column). A major confidence is achieved when $\ell=3$, but it is not constant due to the PDF shape. If the distribution was one-dimensional, at least $75 \%$ of the data would be accurately modeled, despite the density of the measurements. Although this result is not directly applicable to multivariate distributions, it includes a representative set of observed values [24]. In addition, more than $95 \%$ data on average is inside the $3 \sigma$ uncertainty impedance as a result of the proposed counting approach.

The usual suitable trade-off sets a 95\% confidence level. This compromise can be used for estimating the $\ell$ value by solving $p(\ell)=0.95$. Nevertheless, the approximated parameter should be near the real number because the observed sample number $N$ is very large.

Figures 8 and 9 show the T-monopole antenna case $\widehat{\Omega}_{Z}^{95 \%}$ ellipse and the investigated scenarios (data + talk, data, and talk modes). The semiaxis magnitudes ( $a$ and $b$ ) presented

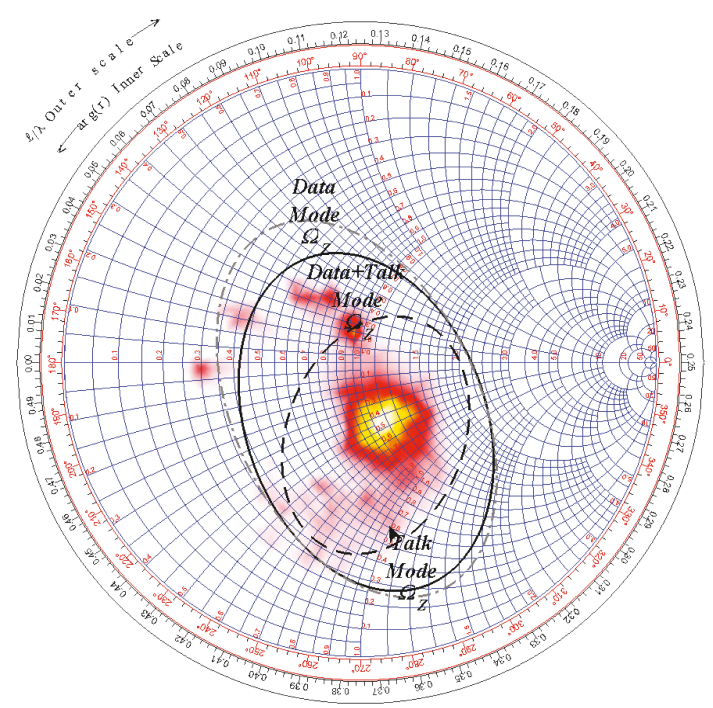

FIgURE 8: Comparison of talk, data, and final uncertainty impedance ellipse in UTRA/FDD LTE B8 band for a Tmonopole antenna.

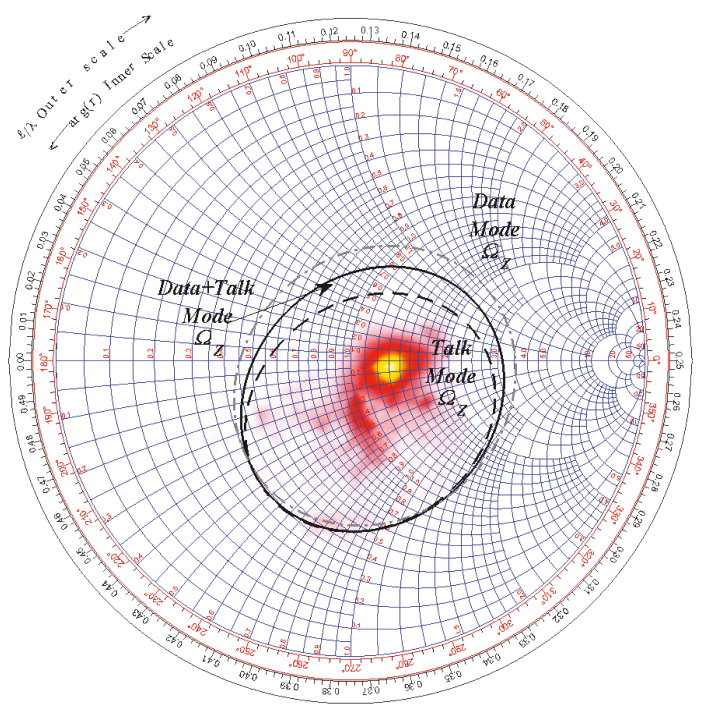

FIgURE 9: Comparison of talk, data, and final uncertainty impedance ellipse in UTRA/FDD LTE B3 band for a Tmonopole antenna.

in Table 1 are computed experimentally with an estimated 95\% confidence level.

One of the main applications of such ellipses in matching issues is the computation of real reconfigurable tuning unit coverage areas. The criteria to design adaptive impedance networks cover the whole Smith chart space according to the classical point of view. In fact, the non-idealities introduced by circuit elements (switches, capacitors, and PIN-diodes) reduce the coverage and make the development to be a difficult task. The impedance ellipse can be taken as a starting point to focus the tuning unit design onto that area, which may result in more simple matching networks. 
TABLE 2: Coverage factor in practical automatic tuning units.

\begin{tabular}{lccc}
\hline & Cantor & Descartes & T-monopole \\
\hline UTRA/FDD LTE B8 & 0.091804 & 0.134487 & 0.240352 \\
UTRA/FDD LTE B3 & 0.034307 & 0.123172 & 0.197955 \\
\hline
\end{tabular}

Indeed, the coverage factor reduction can be evaluated with the aid of the proposed method. Let us think in the ideal tuning unit which covers all the Smith chart and an optimized network which fulfils the actual requirement. Then, the coverage factor $\left(\eta_{Q}\right)$ is the ratio of the areas of the total smith chart, and the uncertainty impedance ellipse

$$
\eta_{Q}=\frac{\pi a b}{\pi r_{\mathbb{S C}}^{2}}=a b
$$

The numerical evaluation (included in Table 2) shows that only $24 \%$ of the coverage is actually required in the worst case to design the adaptive impedance matching network.

\section{Conclusion}

User interaction effects on portable antenna input impedance can be quantified in geometrical terms using a novel framework which is applicable to any structure, coupling objects, and frequency band. This technique relies upon the mathematical support of the experimental statistical probability density functions for the reflection coefficient, which is estimated by means of stochastic magnitudes (the covariance matrix) and the so-called uncertainty impedance ellipse. Its main parameters (center, major and minor axes, and rotation angle) allow engineers to know with certain confidence level the set of feasible impedances which could have an antenna and, thus, to evaluate dynamic mismatching. Furthermore, the method has been applied to three structures predicting some trends in the antenna impedance. This algorithm is a powerful tool for making an accurate choice among possible portable antenna candidates and for reducing coverage requirements on impedance tuning networks.

\section{Conflicts of Interest}

The authors declare that there is no conflict of interest regarding the publication of this paper.

\section{References}

[1] I. Syrytsin, S. Zhang, G. Pedersen, K. Zhao, T. Bolin, and $Z$. Ying, "Statistical investigation of the user effects on mobile terminal antennas for $5 \mathrm{G}$ applications," IEEE Transactions on Antennas and Propagation, vol. PP, no. 99, p. 1, 2017.

[2] M. Okoniewski, M. A. Stuchly, and A. Study, "A study of the handset antenna and human body interaction," IEEE Transactions on Microwave Theory and Techniques, vol. 44, no. 10, pp. 1855-1864, 1996.

[3] M. A. Jensen and Y. Rahmat-Samii, "EM interaction of handset antennas and a human in personal communications," Proceedings of the IEEE, vol. 83, no. 1, pp. 7-17, 1995.
[4] M. Pelosi, O. Franek, M. B. Knudsen, M. Christensen, and G. F. Pedersen, "A grip study for talk and data modes in mobile phones," IEEE Transactions on Antennas and Propagation, vol. 57, no. 4, pp. 856-865, 2009.

[5] J. Holopainen, O. Kivekas, J. Ilvonen, R. Valkonen, C. Icheln, and P. Vainikainen, "Effect of the user's hands on the operation of lower UHF-band mobile terminal antennas: focus on digital television receiver," IEEE Transactions on Instrumentation and Measurement, vol. 53, no. 3, pp. 831-841, 2011.

[6] K. R. Boyle, Y. Yuan, and L. P. Ligthhart, "Analysis of mobile phone antenna impedance variations with user proximity," IEEE Transactions on Antennas and Propagation, vol. 55, no. 2, pp. 364-372, 2007.

[7] M. Pelosi, O. Franek, M. B. Knudsen, G. F. Pedersen, and J. B. Andersen, "Antenna proximity effects for talk and data modes in mobile phones," IEEE Antennas and Propagation Magazine, vol. 52, no. 3, pp. 15-27, 2010.

[8] J. Krogerus, J. Toivanen, C. Icheln, and P. Vainikainen, "Effect of the human body on total radiated power and the 3-D radiation pattern of mobile handsets," IEEE Transactions on Instrumentation and Measurement, vol. 56, no. 6, pp. 23752385, 2007.

[9] L. K. Warne K. S. Lee et al., "Statistical properties of linear antenna impedance in an electrically large cavity," IEEE Transactions on Antennas and Propagation, vol. 51, no. 5, pp. 978-992, 2003.

[10] D. Schnelle and R. E. Wetmore, "Evaluation of antenna and receiver mismatch effects on DTV reception," IEEE Transactions on Broadcasting, vol. 48, no. 4, pp. 365-369, 2002.

[11] P. L. Carro, J. de Mingo, P. Garcia-Ducar, and C. Sanchez, "Performance degradation due to antenna impedance variability in DVB-H consumer devices," IEEE Transactions on Consumer Electronics, vol. 56, no. 2, pp. 1153-1159, 2010.

[12] J. de Mingo and A. Valdovinos, "An RF electronically controlled impedance tuning network design and its application to an antenna input impedance automatic matching system," IEEE Transactions on Microwave Theory and Techniques, vol. 52, no. 2, pp. 489-497, 2004.

[13] C. Hoarau, N. Corrao, J.-D. Arnould, P. Ferrari, and P. Xavier, "Complete design and measurement methodology for a tunable RF impedance-matching network," IEEE Transactions on Microwave Theory and Techniques, vol. 56, no. 11, pp. 2620-2627, 2008.

[14] E. L. Firrao, A.-J. Annema, and B. Nauta, "An automatic antenna tuning system using only RF signal amplitudes," IEEE Transactions on Circuits and Systems II: Express Briefs, vol. 55, no. 9, pp. 833-837, 2008.

[15] A. R. Webb, Statistical Pattern Recognition, John Wiley and Sons, Inc., Chichester, England, 2002.

[16] B. Manimegalai, S. Raju, and V. Abhaikumar, "A multifractal cantor antenna for multiband wireless applications," IEEE Antennas and Wireless Propagation Letters, vol. 8, no. 1, pp. 359-362, 2009.

[17] D.-C. Chang, B.-H. Zeng, and J.-C. Liu, "CPW-fed circular fractal slot antenna design for dual-band applications," IEEE Transactions on Antennas and Propagation, vol. 56, no. 12, pp. 3630-3636, 2008.

[18] K.-L. Wong, G.-Y. Lee, and T.-W. Chiou, "A low-profile planar monopole antenna for multiband operation of mobile handsets," IEEE Transactions on Antennas and Propagation, vol. 51, no. 1, pp. 121-125, 2003. 
[19] Radio Transmission and Reception (Release 10), 2011, 3 GPP TS 45.005 .

[20] R. L. Johnson, "Elliptical error statistics for radiolocation analysis," IEEE Transactions on Aerospace and Electronic Systems, vol. 14, no. 4, pp. 663-665, 1978.

[21] L. R. Paradowski, "Uncertainty ellipses and their application to interval estimation of emitter position," IEEE Transactions on Aerospace and Electronic Systems, vol. 33, no. 1, pp. 126-133, 1997.

[22] S. Geisser, Principles of Modeling Uncertainties in Spatial Data and Spatial Analyses, Chapter 15, CRC-Press, NW, USA, 2010.

[23] M. Reid and B. Szendroi, Geometry and Topology, Cambridge University Press, Cambridge, UK, 2003.

[24] A. W. Marshall and I. Olkin, "Multivariate Chebyshev inequalities," The Annals of Mathematical Statistics, vol. 31, no. 4, pp. 1001-1014, 1960. 


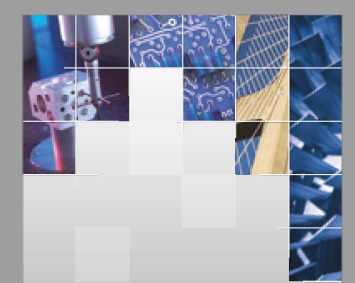

\section{Enfincering}
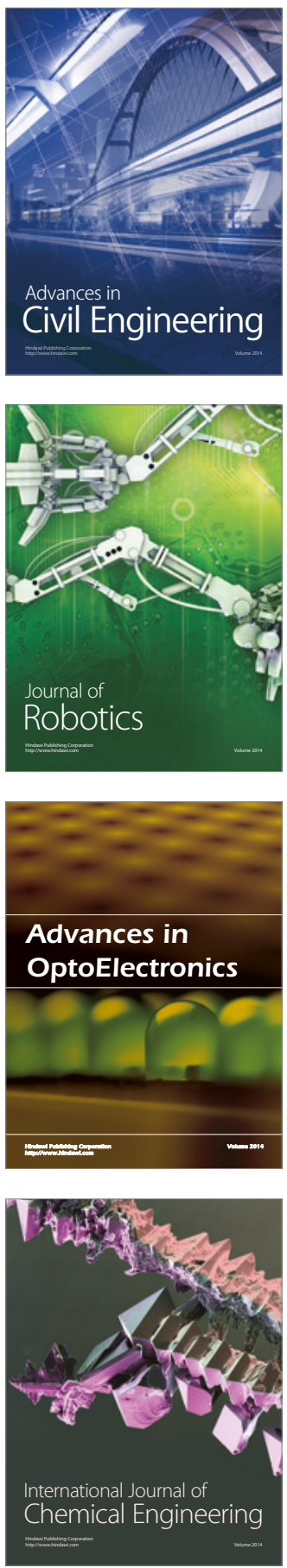

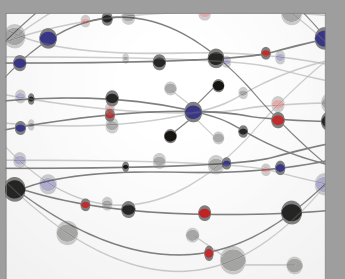

The Scientific World Journal

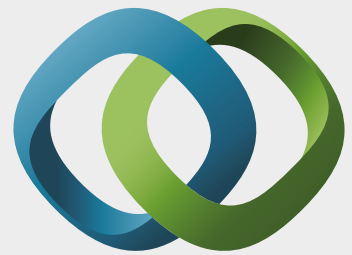

\section{Hindawi}

Submit your manuscripts at

https://www.hindawi.com
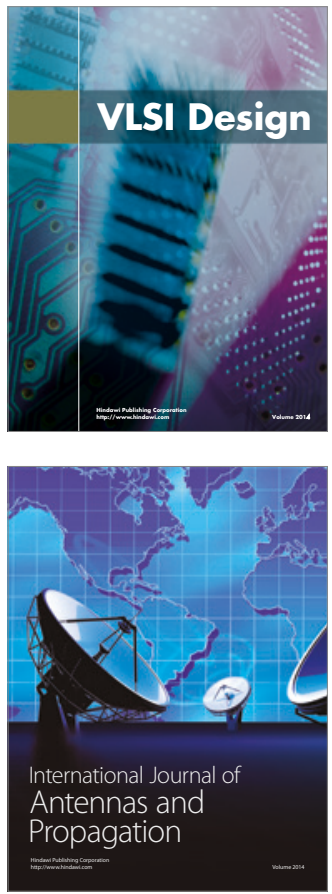

\section{Rotating}

Machinery
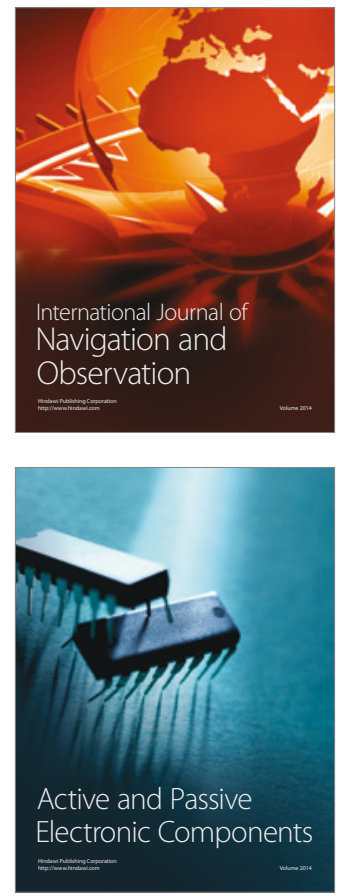
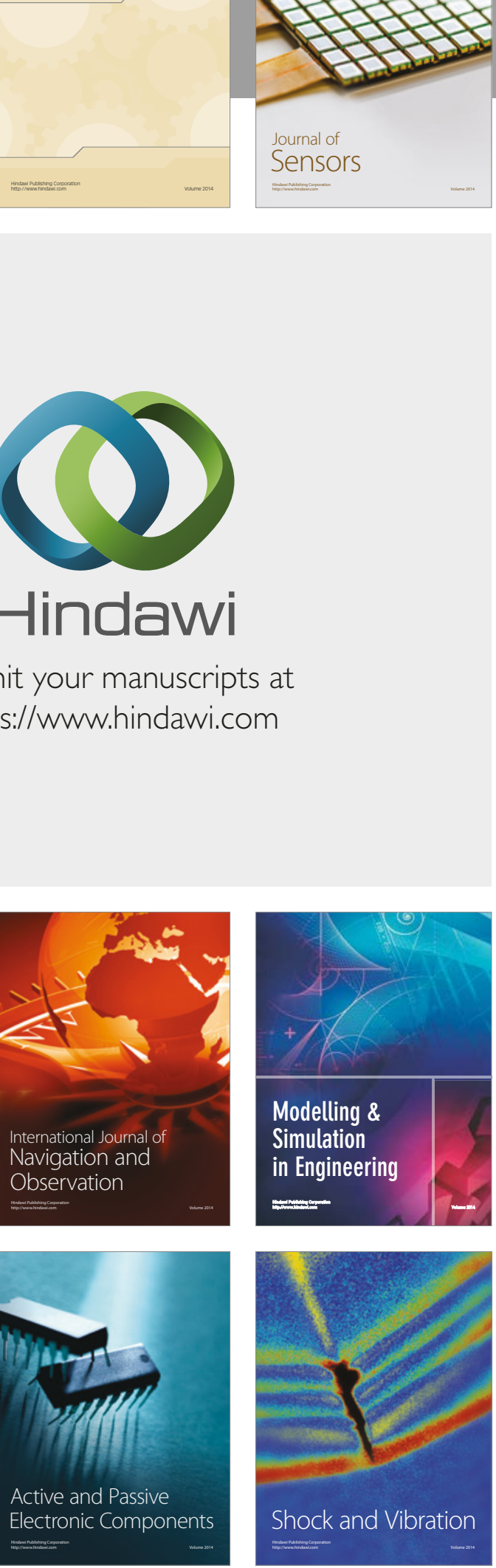
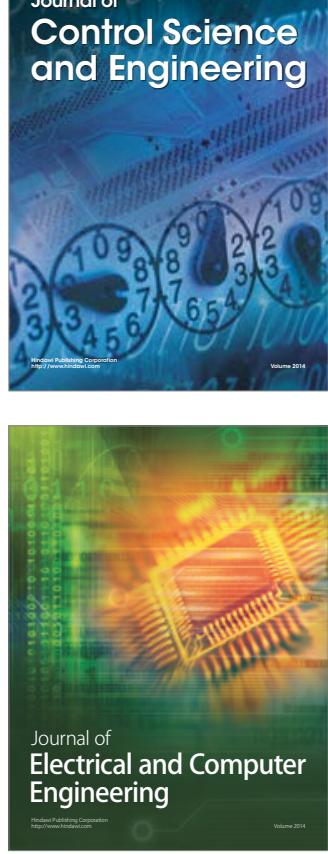

Distributed

Journal of

Control Science

and Engineering
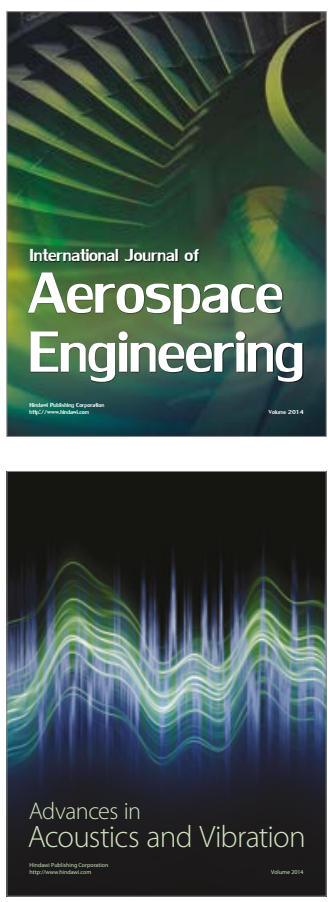

Sensor Networks 